\title{
Instability Risk and Beam Profile Variation in Optical Ring Resonator due to Thin Gradient Index Lens
}

\author{
Khalid Ali Khan ${ }^{1, *}$, Suleyman Malikmyradovich Nokerov ${ }^{2}$ \\ ${ }^{1}$ Department of Electrical and Computer Engineering, Mettu University, Mettu, Ethiopia \\ ${ }^{2}$ Oguz Han Engineering and Technology University of Turkmenistan, City Ashgabat, Turkmenistan \\ Received 15 July 2020; received in revised form 09 October 2020; accepted 15 December 2020 \\ DOI: https://doi.org/10.46604/peti.2021.6551
}

\begin{abstract}
In this study, a simple ring resonator model in presence of thin gradient index (GRIN) lens is investigated to characterize the optical beam maginification quality beyond its traditional modalities. This model allow us to vary and control the limit of resonator stability more significantly.It consist of two folding arms and each arm can be realized by its cavity components. Insertation of thin GRIN lens ( thickness $<9.3 \mathrm{~mm}$ ) in ring resonator, mainly in between first folding range gives the magnified output beams and meets the beam expander feature for the laser. Variation of GRIN lens thickness (L) is an emphatic and influencing parameter than its refractive index (n) to disturb the resonator stability. Resonator stability in Tangential (T) plane is relatively more sensitive than sagittal (S) plane. Vigorous magnification in optical beam size at the end of output range in a cavity is the noticeable consequences because of GRIN lens.
\end{abstract}

Keywords: optical ring resonator, GRIN lens, resonator stability, ABCD matrix, beam propagation microstrip

\section{Introduction}

Actually, optical resonator or cavity with different structure along with their gain is globally known as the integral part of laser and light interferometer. Wide application of resonator such as in quantum electrodynamic [1-2], enhancement of circulating power in resonator [3], in high-sensitivity laser spectroscopy and sensing [4-7], optical switching [8], photonic biosensor [9], in communication system [10] is gaining research popularity in physicists and engineers omnidirectionally. John E. Heebner et al. [11] has also developed the ring resonator theory for the measurement of optical transmission characteristic.But, historically, Fabry-Perot interferometer was the first optical resonator [12-14]. Other than it, Boyd et al. [15-16] modified and modelled the Fabry-Perot resonator with spherical mirrors first. Even- though, several decades earlier, explanation and mathematical expression for Gaussian laser beam propagation has been given by Fox [17], Pierce [18], Goubau [19], Kogelnik [20] and by others. However, complete derivation for LASER resonator to achieve the stability is available in [21]. The light rays that bounce back and forth in between the resonator shows the focusing mechanism.

This paper presents a modified version of optical ring resonator supported by thin gradient index (GRIN). The Presence of GRIN lens improves output performance of the resonator including laser pumping, beam magnification and focusing mechanism under its proper parametric adjustment. Table 1 lists formulated ABCD matrix for used elements [22-23]. The Application of GRIN lens draws the novel anxiety into the possibilities and limitations of resonator stability and on its beam

* Corresponding author. E-mail address: sts.khalid@gmail.com

Tel.: +251-954591921 
magnification property [24]. ABCD matrix has been also used to analyze the cavity. Resonator stability is decided if the condition is satisfied:

$$
-1 \leq \frac{A+D}{2} \leq+1
$$

Consequently, light ray trajectory is close and bounded to optical axis after multiple number of round-trips. In ring resonator, round trip matrix can be calculated by multiplying the elemental ABCD matrix from reference element to first element of the model then after from last element to an element next to the reference.

Table 1 Elements and its ABCD matrix

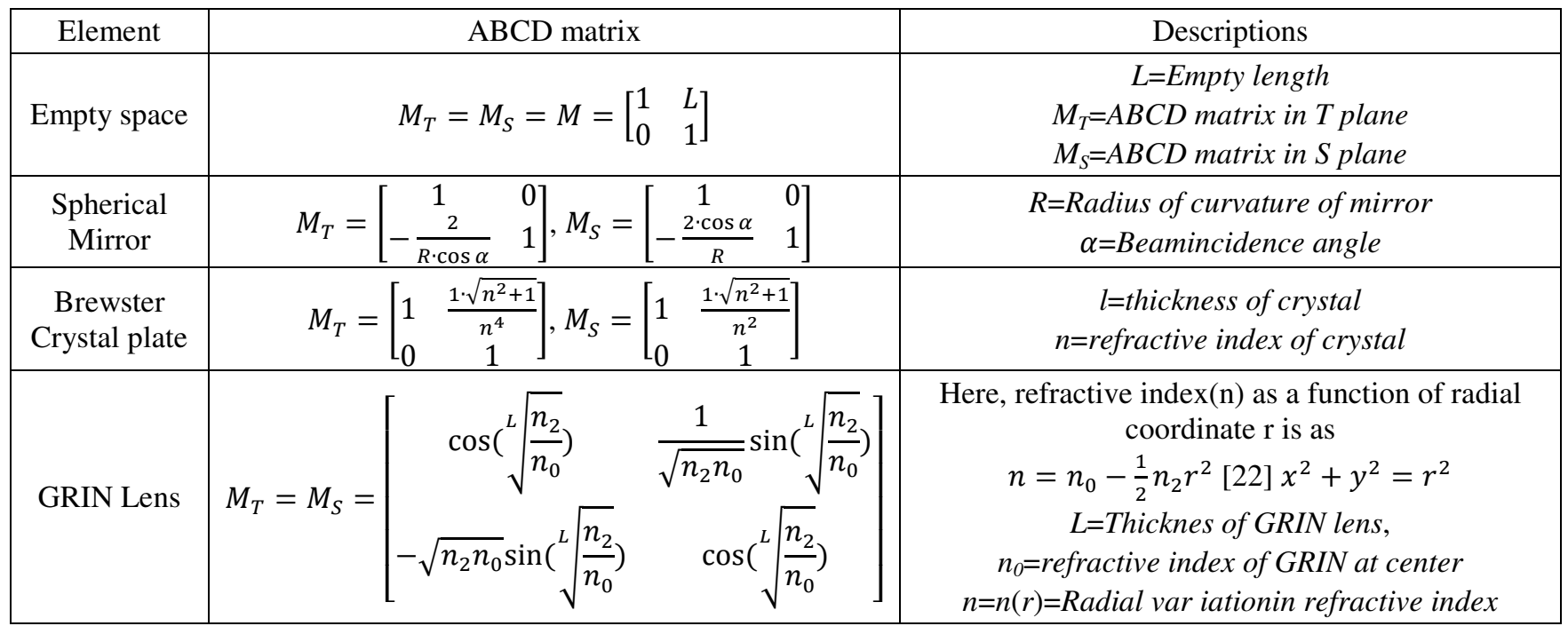

\section{Modelling of Ring Resonator}

The major components (elements) and schematic model of simple ring resonator has been shown in Fig. 1. It is made up of two spherical mirrors (M1, M2), one Brewster crystal plate (Cr1) and with a single piece of radial gradient index (GRIN) lens (F1). Here, thin $\mathrm{Cr} 1$ and thin GRIN lens (F1) has been used to maintain the resonator stability.In fact, total length $\{1300(L 0)+75(d 1)+1.262 \cdot 1.3(C r 1)+50(d 2)+0.1 \cdot 1.5(F 1)+35(L 1)=1460.80 m m\}$ is known as the resonator or cavity length and found to be $1460.80 \mathrm{~mm}$. Mathematically, this length of cavity is the sum of indivisual elemental length. By assumption, beam characteristics are evaulauted and tested at 1550 nanometer $(\mathrm{nm})$ wavelength by software simulation process.

The main objective of the $\mathrm{Cr} 1$ in the resonator is to compensate the astigmatism, whereas, addition of GRIN gives optical beam magnification that is justified by its performance. The technical specifications of all components are listed in Table 2 .

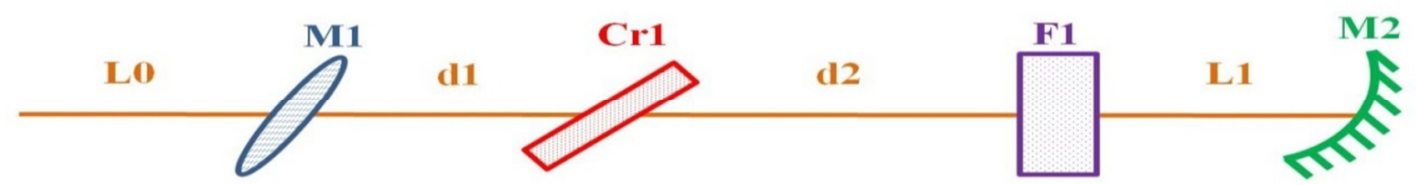

Fig. 1 The Schematic model of ring resonator with GRIN lens

Table 2 The technical specifications of components

\begin{tabular}{|c|c|c|}
\hline S. No & Elements & Specifications \\
\hline 1 & Empty space "L0" (output radius) & $L 0=1300 \mathrm{~mm}$ \\
\hline 2 & Spherical mirror "M1" and M2 & Radius of curvature $(R)=150 \mathrm{~nm}, \alpha=10^{\circ}$ \\
\hline 3 & Empty space "d1", "d2" and "L1" & $d 1=74 \mathrm{~mm}, d 2=50 \mathrm{~mm}$, and $L 1=35 \mathrm{~mm}$ \\
\hline 4 & Brewster crystal plate (Cr1) & Crystal width $(L)=1 \mathrm{~mm}$, Refractive index $(n)=1.3$ \\
\hline 5 & GRIN lens (F1) & Thickness $=0.1 \mathrm{~mm}$, Refractive index $(n)=1.5$ \\
\hline
\end{tabular}


Proper inclination without any deviation is given to the spherical mirrors M1 and M2 so that it is matched with beam incidence angle $(\alpha)$ to ensure maximum capturing of beam. Therefore, the complete round trip matrix (M0) for the proposed cavity model (by considering L0 as reference point) will be as:

$$
M 0=L 0 \cdot M 2 \cdot L 1 \cdot F 1 \cdot d 2 \cdot C r 1 \cdot d 1 \cdot M 1
$$

where L0, L1, M1, M2, F1, d1, d2, Cr1 represent the elemental ABCD matrix that has been calculated as per its specific values and recorded in Table 3 .

Table 3 Elemental ABCD matrix as per mentioned specification

\begin{tabular}{|c|c|c|}
\hline Elements & T-plane matrix & S-plane matrix \\
\hline$L 0$ & {$\left[\begin{array}{cc}1 & 1300 \\
0 & 1\end{array}\right]$} & {$\left[\begin{array}{ll}1 & 1300 \\
0 & 1\end{array}\right]$} \\
\hline$M 2$ & {$\left[\begin{array}{cc}1 & 0 \\
-0.014 & 1\end{array}\right]$} & {$\left[\begin{array}{cc}1 & 0 \\
-0.013 & 1\end{array}\right]$} \\
\hline$L 1$ & {$\left[\begin{array}{cc}1 & 35 \\
0 & 1\end{array}\right]$} & {$\left[\begin{array}{cc}1 & 35 \\
0 & 1\end{array}\right]$} \\
\hline$F 1$ & {$\left[\begin{array}{cc}1 & 0.067 \\
-0.001 & 1\end{array}\right]$} & {$\left[\begin{array}{cc}1 & 0.067 \\
-0.001 & 1\end{array}\right]$} \\
\hline$d 2$ & {$\left[\begin{array}{cc}1 & 50 \\
0 & 1\end{array}\right]$} & {$\left[\begin{array}{cc}1 & 50 \\
0 & 1\end{array}\right]$} \\
\hline$C r 1$ & {$\left[\begin{array}{cc}1 & 0.574 \\
0 & 1\end{array}\right]$} & {$\left[\begin{array}{ll}1 & 0.97 \\
0 & 1\end{array}\right]$} \\
\hline$d 1$ & {$\left[\begin{array}{cc}1 & 74 \\
0 & 1\end{array}\right]$} & {$\left[\begin{array}{cc}1 & 74 \\
0 & 1\end{array}\right]$} \\
\hline$M 1$ & {$\left[\begin{array}{cc}1 & 0 \\
-0.014 & 1\end{array}\right]$} & {$\left[\begin{array}{cc}1 & 0 \\
-0.013 & 1\end{array}\right]$} \\
\hline
\end{tabular}

Hence, by using Eq. (1) and recorded value of individual elemental ABCD matrix as Table 3, the complete round trip matrix in terms of ABCD matrix for proposed resonator in tangential (T) and sagittal (S) plane are respectively (L0 has been taken as reference).

$$
\begin{aligned}
& M_{T}=\left|\begin{array}{cc}
16.77 & -1323.23 \\
0.201 & -15.83
\end{array}\right| \\
& M_{S}=\left|\begin{array}{ll}
15.29 & -1246.96 \\
0.189 & -15.36
\end{array}\right|
\end{aligned}
$$

where, $\mathrm{M}_{\mathrm{T}}=$ round trip matrix in $\mathrm{T}$ plane, $\mathrm{M}_{\mathrm{S}}=$ round trip matrix in $\mathrm{S}$ plane

\section{Simulated Results and Analysis}

\subsection{Effect of gradient Index (GRIN) lens on Stability}

Normal stability condition (Eq. (1)) is considered to decide the stability region in tangential (T) as well as in sagittal plane (S). Plotted stability graph for respective GRIN lens-parameters and $\mathrm{Cr} 1$ plate parameters confine the restricted boundary condition under which back - forth optical pulse oscillation takes place without outward bouncing in a cavity. Simulated graph as in Fig. 2 illustrates that resonator stability is very sensitive toward GRIN lens thickness. A small increment in thickness variation may lose the system stability. For proposed model, thickness should be less than $0.38 \mathrm{~mm}$ in order to sustain stability.

Fig. 3 (a) and Fig. 3(b) are the stability variation plot with respect to refractive index( $n$ ) variation of GRIN lens. This can be easily understood that refractive index variation of GRIN lens does not alter the state of stability in both plane observation 


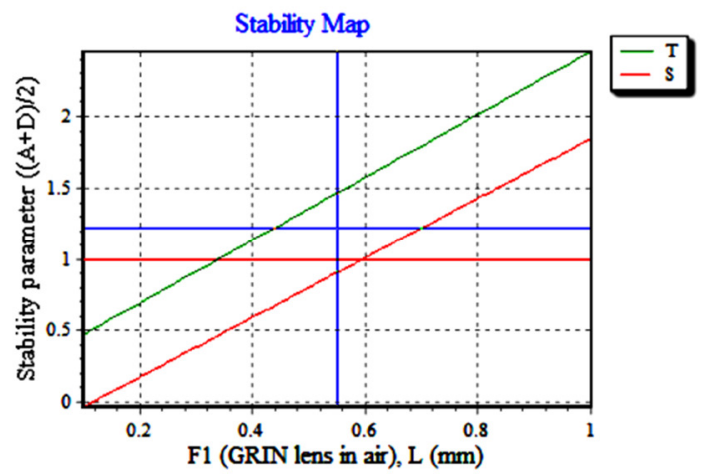

Fig. 2 Stability dependency on GRIN length (L)

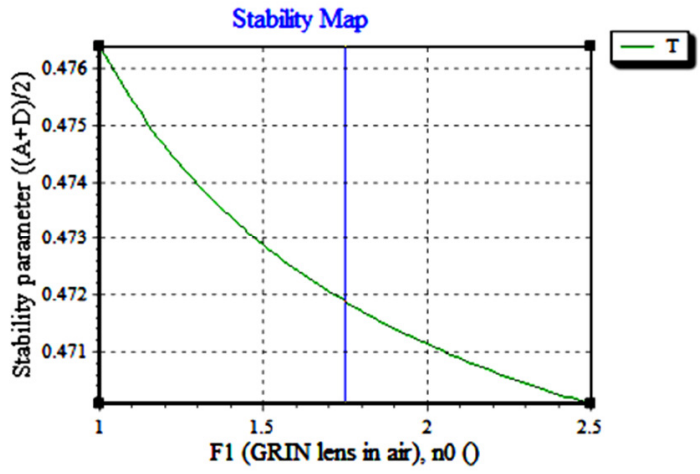

(a) Stability dependency on GRIN refractive index in T plane

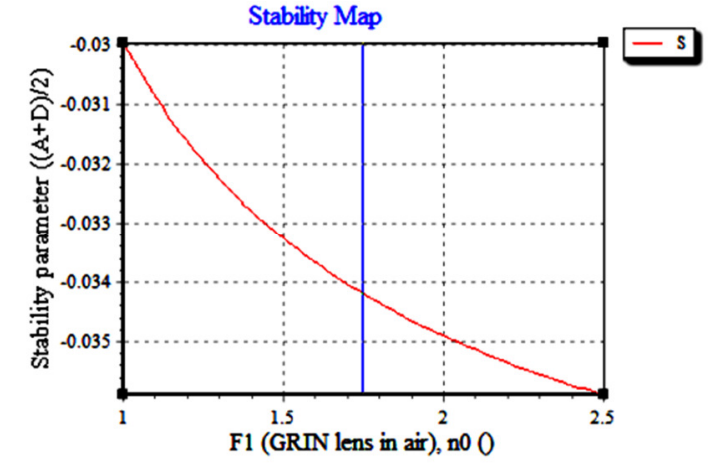

(b) Stability dependency on GRIN refractive index in S plane

Fig. 3 Stability variation graph with variation of GRIN refractive index(n)

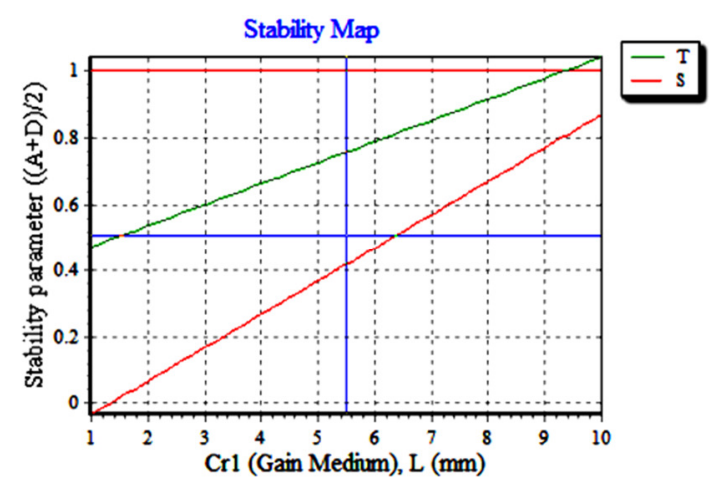

(a) Stability under $\mathrm{Cr} 1$ length (stability continues, if $\mathrm{Cr} 1$ length $<9.3 \mathrm{~mm}$ ) in presence of GRIN

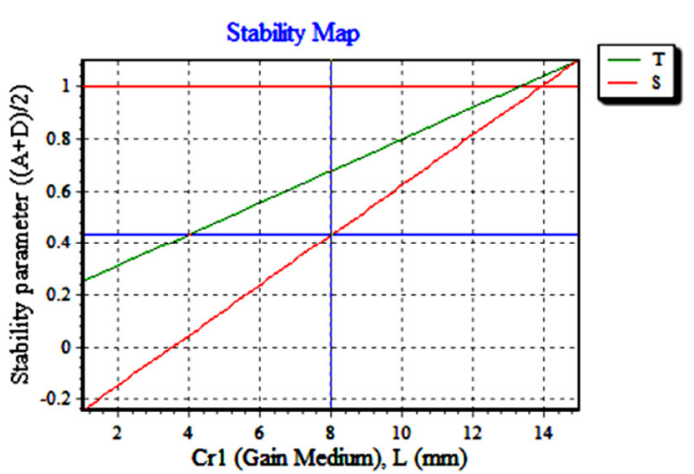

(b) Stability under $\mathrm{Cr} 1$ length (stability continues, if $\mathrm{Cr} 1$ length $<13.43 \mathrm{~mm}$ ) in absence of GRIN

Fig. 4 Impact of Brewster crystal thickness (L) on stability variation

$(\mathrm{T}, \mathrm{S})$. Because slight and unpredictable stability variation is found in T plane (From 0.746 to 0.741 ) as well as in $\mathrm{S}$ plane (from -0.030 to -0.035 ) with respect to large variation in refractive index starting from 1 to 2.5 .

However, $\mathrm{Cr} 1$ thickness play a vital role in stability governance. Here, it is more interesting to note that $\mathrm{Cr} 1$ shows the satisfactory stability performance for both planes, if and only if,

$$
\begin{aligned}
& \text { thickness of } C r 1 \leq 9.32 \mathrm{~mm} \text { (in presence of GRIN) } \\
& \text { thickness of } C r 1 \leq 13.43 \mathrm{~mm} \text { (in absence of GRIN) }
\end{aligned}
$$

Otherwise, resonator may lose stability. Therefore, present of GRIN lens in modelled resonator brings the resonator close to instability region. Fig. 4(a) and Fig. 4(b) illustrates the Cr1 thickness limitation to support stability of resonator.

Furthermore, as far as the refractive index (n) of Cr1 is concerned, its variation does not change the state of stability more significantly, either in the presence or the absence of GRIN. Almost similar plot as drawn in Fig. 5(a) and Fig. 5(b) reveals that 
a slight change in stability is observed if refractive index (n) changes and goes only up to 2.5, afterwards, stability becomes constant in both plane $(\mathrm{T}, \mathrm{S})$. It is noteworthy to clear that stability region in between -0.1 to +0.5 in presence of GRIN lens or in between -0.30 to +0.30 in absence GRIN shows that change in refractive index (n) of Cr1 does not affect seriously.

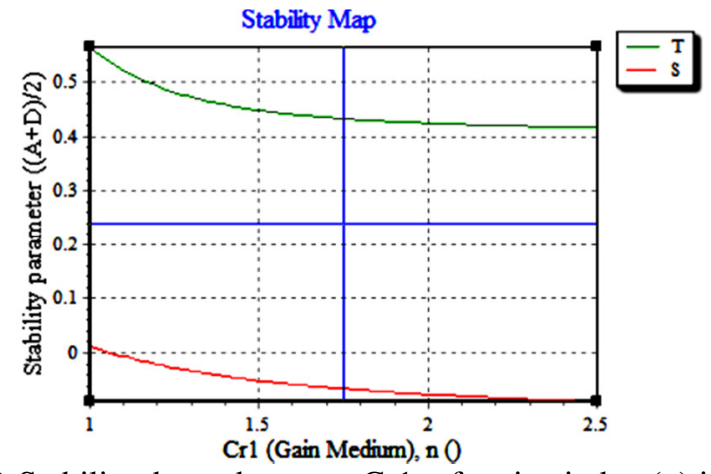

(a) Stability dependency on Cr1 refractive index (n) in presence of GRIN lens

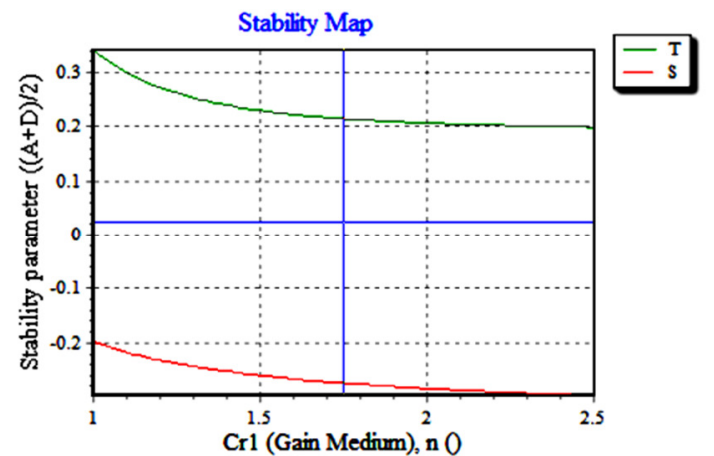

(b) Stability dependency on $\mathrm{Cr} 1$ refractive index (n) in absence of GRIN lens

Fig. 5 Impact of refractive index (n) of Brewster crystal on stability variation

\subsection{Gaussian beam propagation and beam magnification}

In previous section, it was proved that stability of the cavity in both plane (T and S) does not depend upon refractive index (n) of GRIN lens(F1) and refractive index (n) of Brewster crystal plate (Cr1). The ABCD matrix for respective, spherical mirrors, Brewster plate and GRIN lens are independent to its position inside the cavity except empty space. However. This ABCD matrix is useful to calculate the beam parameter throughout the cavity.

In most cases, it pre-assumed that laser beam propagation has ideal Gaussian intensity profile, even-though it is not true in real time. However, we will assume theoretical properties of Gaussian beam with quality factor, $\mathrm{M}^{2}=1$ (It is known as " $\mathrm{M}$ squared" factor). The beam radius variation in entire cavity in presence and in absence GRIN lens are shown in Fig. 6(a) and Fig. 6(b) respectively.

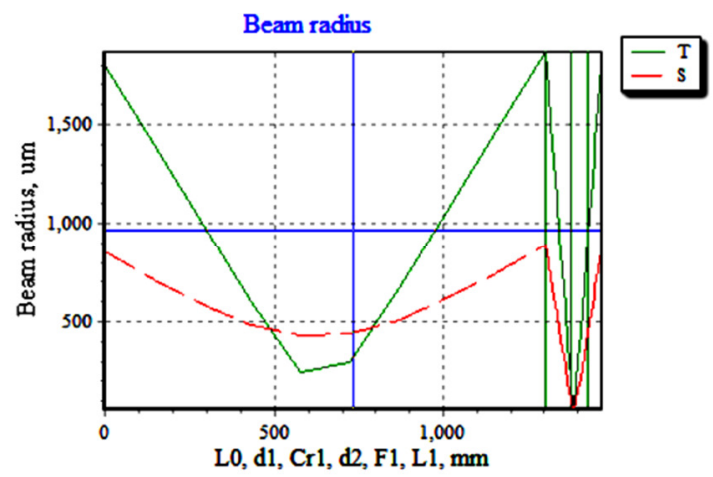

(a) Beam radius throughout the cavity in presence of GRIN lens

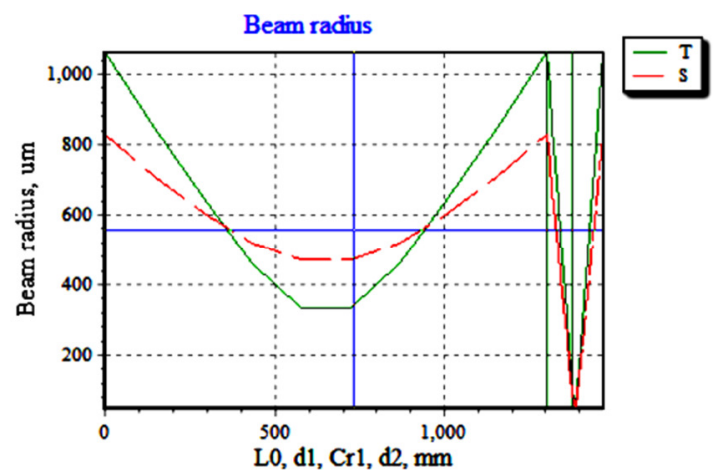

(b) Beam radius throughout the cavity in absence of GRIN lens

Fig. 6 Beam radius vartiaon throughout the cavity

Here, it is more interesting to comment that there is abrupt contraction in beam radius in between both spherical mirrors (M1, M2) in both cases. Larger beam radius is found at the interface of spherical mirrors and beam exit end (L0=0) of the cavity. Additionally, sudden beam increment can be also seen at the Brewster crystal surface in tangential plane. Because when beam passes through it, refracted beam size in tangential plane increased due to increase in refractive index. Therefore, it is clear from Fig. 6(a) and Fig. 6(b) that inclusion of GRIN lens in between Brewster crystal plate and M2 (it is also called first folding range) in ring resonator provides the magnified beam to the respective output range (L0). Referring to Fig. 6(a), it is found that minimum beam radius across L0 is 243 micrometer (um) that is found at $577.77 \mathrm{~mm}$ location. Hereafter, a small increment in beam radius exists up to $722.22 \mathrm{~mm}$ distance and finally it becomes $295.43 \mathrm{um}$. 
Nevertheless, in absence of GRIN lens, the beam variation in between $577.77 \mathrm{~mm}$ to $722.22 \mathrm{~mm}$ length across L0 is almost zero and found constant. However, this segment $(577.77 \mathrm{~mm}$ to $722.22 \mathrm{~mm}$ ) shows a drastic change in wavefront curvature radius in $\mathrm{S}$ plane. Thus, recorded data shows that (in $\mathrm{S}$ plane in presence of GRIN)

$$
\begin{aligned}
& \mid \text { wave front radius }\left.\right|_{\text {at } L 0=577.77 m m}=-2396.46 \mathrm{~mm} \\
& \mid \text { wave front radius }\left.\right|_{\text {at } L 0=722.22 \mathrm{~mm}}=+1537.95 \mathrm{~mm}
\end{aligned}
$$

Therefore, Fig. 7(a) and Fig. 7(b) reveals that in S plane, abrupt and large transition in wavefront radius between two point predicts slow and small radius variation at that point and vice versa.it must be also recorded that the contraction of beam radius yields negative and smaller wavefront curvature radius, whereas beam radius expansion generates positive and larger wavefront curvature radius.

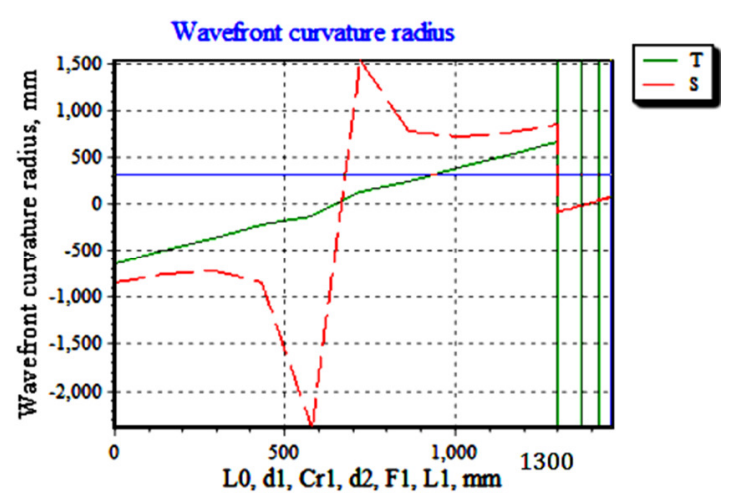

(a) Wavefront radius variation throughout the cavity in presence of GRIN lens

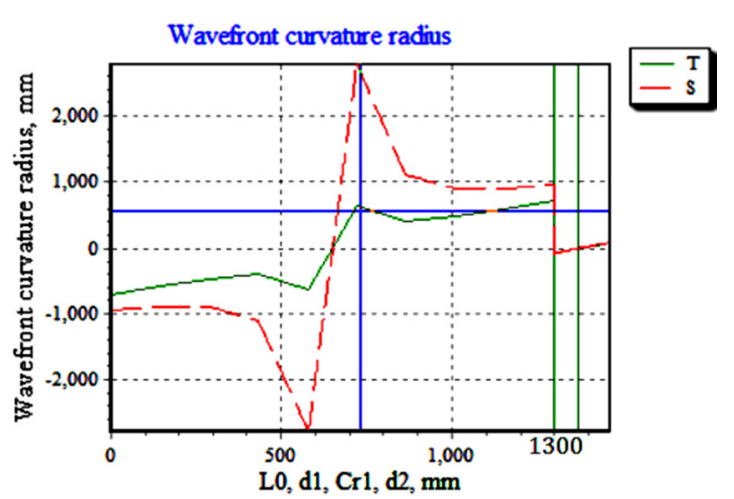

(b) Wavefront radius variation throughout the cavity in absence of GRIN lens

Fig. 7 Wavefront radius variation throughout the cavity

Alternatively, sudden and unexpected wavefront radius transition can be also observed at L0=1300 mm (in S plane) at which wavefront radius of $+860.81 \mathrm{~mm}$ becomes to $-83.54 \mathrm{~mm}$.

Relatively, cavity without GRIN lens that produces smaller beam spot across L0, generates larger wavefront curvature radius corresponding to it. For example, beam wavefront radius (in S plane)

$$
\begin{aligned}
& \mid \text { wave front radius }\left.\right|_{\text {at } L 0=577.77 \mathrm{~mm}}=+2794.87 \mathrm{~mm} \\
& \mid \text { wave front radius }\left.\right|_{\text {at } L 0=722.22 \mathrm{~mm}}=+2794.87 \mathrm{~mm}
\end{aligned}
$$

Similarly, at the interface of M1 $(\mathrm{L} 0=1300 \mathrm{~mm})$ another abrupt change in wavefront radius is found from $+952.51 \mathrm{~mm}$ to $-82.77 \mathrm{~mm}$.

\section{Conclusions}

In this paper, we have investigated the impact of GRIN lens on resonator stability and beam characteristics in optical ring resonator. Designed resonator has the cavity length and inter-mode-beat frequency of $1460.80 \mathrm{~mm}$ and $205.226 \mathrm{MHz}$ respectively. Inter-mode-beat frequency reflects the pulse repetition rate of $205.226 \mathrm{MHz}$ that estimates the theoretical round-trip distance of $1461.80 \mathrm{~mm}$ inside the cavity. A concise comparison with the commonly used ring resonator with same cavity length is also performed.

Referring to Fig. 3 and Fig. 4, it can be concluded that placing of thin GRIN lens in first folding range (in between Cr1 to M2) achieves the beam size magnification across output range (L0) without altering the stability of ring resonator. Larger beam 
magnification (around 1.7 times) is recorded at the end of L0 in T plane. Thickness variation of GRIN lens disturb the resonator stability more vigorously in $\mathrm{T}$ plane if it is not designed properly. Nevertheless, its application as beam expander may correspond easy portability with other optical devices rather than Keplerian and Galilean beam expander (Keplerian and

Galilean beam expander are unportable for laser beam)

\section{Conflicts of Interest}

The authors declare no conflict of interest.

\section{References}

[1] Y. Jiang, Y. Mei, Y. Zou, Y. Zuo, and S. Du, "Intracavity Cold Atomic Ensemble with High Optical Depth," Review of Scientific Instruments, vol. 90, no. 1, pp. 013105-1-013105-7, January 2019.

[2] J. Ye, C. J. Hood, T. Lynn, H. Mabuchi, D. W. Vernooy, and H. J. Kimble, "Quantum Manipulation and Measurement of Single Atoms in Optical Cavity QED," IEEE Transactions on Instrumentation and Measurement, vol. 48, no. 2, pp. 608-612, May 1999.

[3] A. Mills, S. Zhdanovich, M. X. Na, F. Boschini, E. Razzoli, M. Michiardi, et al., "Cavity-Enhanced High Harmonic Generation for Extreme Ultraviolet Time and Angle-Resolved Photoemission Spectroscopy,” Review of Scientific Instruments, vol. 90, no. 8, pp. 083001-1-083001-9, August 2019.

[4] B. Liu, H. Zhou, L. Liu, X. Wang, M. Shan, P. Jin, and Z. Zhong, “An Optical Fiber Fabry-Perot Microphone Based on Corrugated Silver Diaphragm,” IEEE Transactions on Instrumentation and Measurement, vol. 67, no. 8, pp. 1994-2000, August 2018.

[5] C. Zhu, Y. Zhuang, Y. Chen, and J. Huang, "A Liquid-Level Sensor Based on a Hollow Coaxial Cable Fabry-Perot Resonator with Micrometer Resolution," IEEE Transactions on Instrumentation and Measurement, vol. 67, no. 12, pp. 2892-2897, December 2018.

[6] A. Cygan, P. Wcisło, S. Wojtewicz, G. Kowzan, M. Zaborowski, D. Charczun, et al., "High-Accuracy and Wide Dynamic Range Frequency-Based Dispersion Spectroscopy in an Optical Cavity," Optics Express, vol. 27, no. 15, pp. 21810-21821, July 2019.

[7] G. Gagliardi and H.-P. Loock, Cavity-Enhanced Spectroscopy and Sensing. Springer Series in Optical Sciences 179 , Heidelberg: Springer-Verlag Berlin Heidelberg, 2014.

[8] V. Van, T. A. Ibrahim, K. Ritter, P. P. Absil, F. G. Johnson, R. Grover, et al., "All Optical Nonlinear Switching in GaAs-AlGaAs Microring Resonators,” IEEE Photonics Technology Letters., vol. 14, no. 1, pp. 74-76, January 2002.

[9] R. W. Boyd and J. E. Heebner, "Sensitive Disk-Resonator Photonic Biosensor," Applied Optics, vol. 40, no. 31, pp. 5742-5747, December 2001.

[10] B. E. Little, S. T. Chu, H. A. Haus, J. Foresi, and J.-P. Laine, "Micro-Ring Resonator Channel Dropping Filters," Journal of Lightwave Technology, vol. 15, no. 6, pp. 998-1005, April 1997.

[11] J. E. Heebner, V. Wong, A. Schweinsberg, R. W. Boyd, and D. J. Jackson, "Optical Transmission Characteristics of Fiber Ring Resonators,” IEEE Journal of Quantum Electronics, vol. 40, no. 6, pp. 726-730, June 2004.

[12] R. H. Dicke, Molecular Amplification and Generation Systems and Methods, U. S. Patent, 2 851 652, September 9, 1958.

[13] A. M. Prokhorov, "Molecular Amplifier and Generator for Submillimeter Waves," Journal of Experimental and Theoretical Physics, vol. 7, no. 6, pp.1658-1659, June 1958.

[14] A. L. Schawlow and C. H. Townes, "Infrared and Optical Masers," Physical Review., vol. 112, no. 6, pp.1940-1949, December 1958.

[15] G. D. Boyd and J. P. Gordon, "Confocal Multimode Resonator for Milli Meter Through Optical Wavelength Masers," The Bell System Technical Journal, vol. 40, no. 2, pp. 489-508, March 1961.

[16] G. D. Boyd and H. Kogelnik, "Generalized Confocal Resonator Theory,” The Bell System Technical Journal., vol. 41, no. 4, pp. 1347-1369, July 1962.

[17] A. G. Fox and T. Li, "Resonant Modes in a Maser Interferometer,” The Bell System Technical Journal., vol. 40, no. 2, pp. 453-488, March 1961.

[18] J. R. Pierce, "Modes in Sequences of Lenses," Proceeding of the National Academy of Sciences of the United States of America, , National Academy of Sciences Press, November 1961, pp. 1808-1813.

[19] G. Goubau, “Optical Relations for Coherent Wave Beams," Electromagnetic Theory and Antennas, New York: Mac Millan, June 1962, pp. 907-918. 
[20] H. Kogelnik, "Imaging of Optical Modes-Resonators with Internal Lenses," The Bell System Technical Journal, vol. 44, no. 3, pp. 455-494, March 1965.

[21] H. Kogelnik and T. LI, "Laser Beams and Resonators," Applied Optics, vol. 5, no. 10, pp.1550-1557, October 1966.

[22] "reZonator Application," www.rezonator.orion-project.org, June 5, 2020.

[23] S. N. B. Tushar, S. D. Nath, J. Akhtar, and M. I. Reja, "Modelling and Analysis of Z- Folded Solid State Laser Cavity with Two Curved Mirrors," International Journal of Microwave and Optical Technology, vol.13, no.3, pp. 244-253, May 2018.

[24] K. A. Khan and S. M. Nokerov, "Analysis of Gaussian Beam Profile and its Amplification in Ring Resonator with Thin Gradient-Index Lens," Modern Means of Communications, Materials of the $25^{\text {th }}$ International Scientific and Technical Conference, Belarusian State Academy of Communications Press, October 2020, pp. 42-44. 